\title{
Quality-of-life trajectories after stereotactic radiosurgery for brain metastases
}

\author{
Adomas Bunevicius, MD, PhD, ${ }^{1}$ Karen Lavezzo, RN, BSN, ${ }^{1}$ Leah Shabo, BS, ${ }^{2}$ \\ Jesse McClure, PharmD, PhD, ${ }^{2}$ and Jason P. Sheehan, MD, PhD' \\ 'Department of Neurological Surgery, University of Virginia Health System; and ${ }^{2}$ School of Medicine, University of Virginia, \\ Charlottesville, Virginia
}

OBJECTIVE Quality of life (QOL) is an important endpoint measure of cancer treatment. The authors' goal was to evaluate QOL trajectories and prognostic value in cancer patients treated with stereotactic radiosurgery (SRS) for brain metastases.

METHODS Patients who underwent Gamma Knife radiosurgery (GKRS) between January 2016 and November 2019 were prospectively evaluated for QOL using the EQ-5D-3L questionnaire before SRS and at follow-up visits. Only patients who had pre-SRS and at least 1 post-SRS QOL assessment were considered.

RESULTS Fifty-four cancer patients underwent 109 GKRS procedures. The first post-SRS visit was at a median of 2.59 months (range 0.13-21.08 months), and the last post-SRS visit was at 14.72 months (range 2.52-45.21 months) after SRS. There was no statistically significant change in the EQ-5D index score $(p=0.539)$ at the first compared with last post-SRS visit. The proportion of patients reporting some problems on the EQ-5D dimension of self-care increased during the course of follow-up from $9 \%$ (pre-SRS visit) to $18 \%$ (last post-SRS visit; $p=0.03$ ). The proportion of patients reporting problems on the EQ-5D dimensions of mobility, usual activities, pain/discomfort, and anxiety/depression remained stable during the course of follow-up $(p \geq 0.106)$. After adjusting for clinical variables, a higher recursive partitioning analysis (RPA) class (i.e., worse prognostic category) was independently associated with greater odds for $E Q-5 D$ index score deterioration $(p=0.050)$. Upfront whole-brain radiation therapy predicted deterioration of the $E Q-5 D$ self-care $(p=0.03)$ and usual activities $(p=0.024)$ dimensions, while a greater number of lesions predicted deterioration of the EQ-5D anxiety/depression dimension $(p=0.008)$. A lower pre-SRS EQ-5D index was associated with shorter survival independently from clinical and demographic variables (OR 18.956, 95\% CI 2.793-128.64; $p=0.003$ ).

CONCLUSIONS QOL is largely preserved in brain metastasis patients treated with SRS. Higher RPA class, upfront whole-brain radiation therapy, and greater intracranial disease burden are independent predictors of post-SRS QOL deterioration. Worse pre-SRS QOL predicts shorter survival. Assessment of QOL is recommended in brain metastasis patients managed with SRS.

https://thejns.org/doi/abs/10.3171/2020.4.JNS20788

KEYWORDS stereotactic radiosurgery; brain metastases; Gamma Knife radiosurgery; quality of life; cancer; survival; oncology

$\mathrm{B}$ RAIN metastases are the most common intracranial tumors in adults, with an incidence rate ranging from 8.3 to 14.3 per 100,000 population. ${ }^{1}$ It is estimated that up to one-third of cancer patients will develop brain metastases at some point during the course of their disease. ${ }^{2,3}$ The incidence of patients presenting with brain metastases is expected to grow, given advancements in cancer therapies leading to longer patient survival and increasing availability of brain MRI for imaging surveillance allowing earlier detection of small and asymptomatic metastatic deposits. ${ }^{4}$

Ionizing radiation plays a central role in the management of intracranial metastases. ${ }^{5,6}$ Stereotactic radiosurgery (SRS) allows precise delivery of radiation to treat intracranial lesions for durable local control, and it is therefore considered a standard of care for brain metastases. ${ }^{7,8}$

ABBREVIATIONS EORTC = European Organization for Research and Treatment of Cancer; FACT-BR = Functional Assessment of Cancer Therapy-Brain; GKRS = Gamma Knife radiosurgery; GPA = Graded Prognostic Assessment; $Q O L$ = quality of life; RPA = recursive partitioning analysis; SRS = stereotactic radiosurgery; WBRT = whole-brain radiation therapy.

SUBMITTED March 13, 2020. ACCEPTED April 22, 2020.

INCLUDE WHEN CITING Published online July 10, 2020; DOI: 10.3171/2020.4.JNS20788. 
SRS can be associated with better preservation of cognitive functioning and quality of life (QOL) when compared with whole-brain radiation therapy (WBRT) ${ }^{9-11}$ Preservation of cognitive functioning is important for maintaining QOL of long-term cancer survivors.

Overall and progression-free survival are important traditional outcome measures that are often used to measure cancer treatment efficacy in clinical trials. Continuous improvement of survival of cancer patients underscores the importance of patient-reported outcomes and QOL. ${ }^{12}$ QOL has prognostic value in cancer patients; consideration of QOL is recommended for cancer treatment guidance, and it is increasingly utilized as an outcome measure in cancer clinical trials. ${ }^{13-15} \mathrm{QOL}$ is also increasingly studied in brain metastasis patients after SRS..$^{16-19}$ Some of the prior studies examining QOL after SRS were limited by small sample sizes, short duration of followup, retrospective study design, and/or a limited number of QOL assessments after the SRS procedure (usually at 1 post-SRS visit). Adequate duration of post-SRS follow-up is important because radiation-induced brain changes are dynamic processes that often take months to develop and cause symptoms. ${ }^{20-22}$

Thus, in the current study of cancer patients undergoing SRS for brain metastases, we sought to evaluate trajectories and predictors of post-SRS QOL. We also explored the prognostic value of pre-SRS QOL for post-SRS outcomes and patient survival. We accessed data that were prospectively collected as part of the national quality registry for SRS initiated by the AANS through the NeuroPoint Alliance. $^{23}$

\section{Methods}

\section{Patients and Procedure}

The registry was deemed as IRB exempt, as it was designed to evaluate the quality of care of brain metastasis patients treated at the University of Virginia Health System with SRS. Informed consent specific to the data collection for this study was not obtained from patients included in the report.

The institutional database at the University of Virginia Health System was queried for patients who were treated with the Gamma Knife radiosurgery (GKRS) for brain metastases between January 2016 and November 2019. Our study protocol followed the AANS NeuroPoint Alliance national quality registry protocol for SRS. We included only those patients who had QOL assessment using the EQ-5D-3L questionnaire before the initial SRS procedure and had at least 1 QOL assessment after SRS. We gathered sociodemographic data (e.g., age, sex, and marital status), clinical data (comorbidities, prior cancer treatments, psychiatric medication use, and presence of extracranial metastases), and relevant GKRS dosimetry parameters. The duration of follow-up was defined the as time (in months) from GKRS until the last follow-up in clinic or death. The Graded Prognostic Assessment (GPA) ${ }^{24}$ score and prognostic class according to recursive partitioning analysis $(\mathrm{RPA})^{25}$ were also estimated. The imaging follow-up duration was defined as the interval (in months) from GKRS until the last available imaging follow-up. Tumor response was defined on brain MRI as stable/decreased or progression based on prior assessment tools. ${ }^{26}$

We used the EQ-5D-3L questionnaire to evaluate QOL at the pre-SRS clinic visit and at all post-SRS clinic visits. Patients completed the EQ-5D-3L questionnaire without the presence of a healthcare provider. All available EQ5D-3L scores at follow-up visits were recorded.

\section{QOL Assessment}

The EQ-5D-3L questionnaire evaluates perceived health status in 5 dimensions of health, including mobility, self-care, usual activities, pain/discomfort, and anxiety/ depression. ${ }^{27}$ Each dimension is rated according to a degree of health state on the day of the assessment. Possible answers for each EQ-5D dimension include no problems (1), moderate problems (2), and severe problems (3), with a greater score being associated with greater perceived problems. The EQ-5D health index is then calculated by using scores on each dimension and can range from 0 to 1 , with a higher index score indicating better QOL. The EQ5D-3L is commonly used in patients with cancer, including patients with metastatic brain tumors. ${ }^{17,18,28}$

The change in EQ-5D index after SRS was estimated by subtracting the EQ-5D index score at the first and last post-SRS visits from the EQ-5D index score at the pre-SRS visit. A minimal clinically important difference of 0.1 was used to categorize post-SRS changes of the health index relative to the pre-SRS level as improvement (change > 0.1 points), no change (from -0.1 to 0.1 points), or worsening $\left(<-0.1\right.$ points) ${ }^{17,18,27,29}$ We also estimated the change of perceived difficulties on all of the EQ-5D dimensions by comparing responses at the pre-SRS visit with responses from the last post-SRS visit on the respective EQ-5D subscales. Patients reporting greater perceived difficulties on the EQ-5D subscales at last post-SRS assessment versus pre-SRS assessment were categorized as worsened, while those who reported better or improved perceived difficulties were deemed as stable.

\section{GKRS Procedure}

GKRS procedures were performed as described previously. ${ }^{30}$ Patients were selected for the GKRS procedure according to existing guidelines considering intracranial tumor burden, number and size of intracranial metastases, functional status, and systemic tumor control. GKRS plans were tailored to the individual patient situation considering size, number, and location of intracranial metastases and prior radiation therapy. All patients underwent GKRS using the Gamma Knife Icon unit (Elekta AB). We used frame-based stereotaxy with the Leksell model G-frame (Elekta AB). The frame was placed under local anesthesia with conscious sedation. Afterward, thin-slice CT scans were acquired and merged with the thin-slice contrast-enhanced T1-weighted brain MR images that were used for treatment planning. Planning MRI was performed within 1 week preceding the SRS procedure. Treatment plans were generated using the GammaPlan software (Elekta $\mathrm{AB})$. GKRS dose planning was performed by a multidisciplinary team that included a neurosurgeon, radiation oncologist, and medical physicist. 


\section{Statistical Analysis}

Statistical analyses were performed using IBM SPSS Statistics for Windows (version 25.0, IBM Corp). For all statistical tests, $\mathrm{p} \leq 0.05$ was considered statistically significant. Categorical data are presented as number (percent) and continuous data as the mean \pm standard deviation and median (minimal value - maximal value).

First, by using the Friedman test, we evaluated changes in QOL after SRS by comparing responses on each of the 5 EQ-5D dimensions and EQ-5D index score across the 3 study visits (before SRS and at the first and last post-SRS visits). Next, using multivariate binary logistic regression analysis, we evaluated predictors of worsening of the EQ$5 \mathrm{D}$ index score and scores on each of the 5 EQ-5D dimensions at the last post-SRS visits in comparison with the pre-SRS assessment. Based on prior studies exploring predictors of post-SRS QOL, ${ }^{17,18}$ we chose age, RPA class, prior WBRT, number of treated lesions, and Karnofsky Performance Scale score as predictor variables for our models. Finally, we used Kaplan-Meier and Cox regression analyses to investigate the association of pre-SRS QOL (pre-SRS EQ-5D score lower vs higher than the median value) with overall survival adjusting for age, marital status, BMI, primary cancer type, RPA class, chemotherapy and/or immunotherapy in the past month, prior WBRT, prior resection, presence of brainstem lesions, number of prior GKRS procedures, number of treated lesions, and EQ-5D index before the SRS.

\section{Results}

\section{Patient and Treatment Characteristics}

Fifty-four cancer patients who underwent 109 GKRS procedures were included in the study (Table 1). The median number of GKRS procedures per patient was 2 (range 1-8). A majority of patients had a GPA score ranging from 1.5 to $3.0(61 \%)$ and an RPA functional class of $2(63 \%)$. The most common primary cancer types were non-small cell lung cancer (37\%) and melanoma (32\%), followed by renal cell carcinoma (7\%), breast cancer (7\%), and colon cancer (4\%). Sixty-nine percent of patients had extracranial metastases, and 52\% received chemotherapy or immunotherapy within 1 month before GKRS. Forty-four percent of patients had prior resection surgery, and $11 \%$ of patients received upfront WBRT. The median number of treated lesions per GKRS session was 2 (range 1-14) (Table 2). Patients with prior WBRT had a greater median number of lesions treated when compared with patients without WBRT histories (4 [range 2-10] vs 1 [range 1-11] $p=0.003)$. The majority of metastatic lesions were in the frontal lobe $(61 \%)$. The median total volume of metastatic lesions at each GKRS session was $1.17 \mathrm{~cm}^{3}$ (range $0.07-20.27 \mathrm{~cm}^{3}$ ). The median prescription dose was $18 \mathrm{~Gy}$ (range 12-32 Gy).

\section{Clinical Outcomes}

Clinical outcomes are presented in Table 3. The mean duration of clinical follow-up was $17.02 \pm 12.05$ months (range 2.23-45.15 months), and the mean duration of imaging follow-up was $14.34 \pm 14.52$ months (range 1-101 months). During the follow-up period, $24 \%$ of patients,
TABLE 1. Demographic and clinical characteristics of the study patients

\begin{tabular}{|c|c|}
\hline Characteristic & Value \\
\hline No. of patients & 54 \\
\hline No. of SRS procedures & 109 \\
\hline \multicolumn{2}{|l|}{ No. of SRS sessions per patient } \\
\hline Mean & $1.94 \pm 1.43$ \\
\hline Median & $2(1-8)$ \\
\hline No. of lesions treated & 357 \\
\hline \multicolumn{2}{|l|}{ Age, yrs } \\
\hline Mean & $65.26 \pm 9.95$ \\
\hline Median & $66(48-82)$ \\
\hline \multicolumn{2}{|l|}{ Sex } \\
\hline Male & $27(50)$ \\
\hline Female & $27(50)$ \\
\hline \multicolumn{2}{|l|}{ Marital status } \\
\hline Married & $43(80)$ \\
\hline Single & $3(6)$ \\
\hline Widowed & $7(13)$ \\
\hline Unknown & $1(2)$ \\
\hline \multicolumn{2}{|l|}{ BMI } \\
\hline Mean & $27.16 \pm 5.89$ \\
\hline Median & $27.6(14.5-44.4)$ \\
\hline Comorbid cardiovascular disease & $34(63)$ \\
\hline Comorbid pulmonary disease & $15(28)$ \\
\hline Other comorbidity & $40(74)$ \\
\hline History of mental disorders & $7(13)$ \\
\hline Psychiatric medication use & $6(11)$ \\
\hline \multicolumn{2}{|l|}{ RPA class } \\
\hline 1 & $19(35)$ \\
\hline 2 & $34(63)$ \\
\hline 3 & $1(2)$ \\
\hline \multicolumn{2}{|l|}{ GPA score } \\
\hline 0.5 & $1(2)$ \\
\hline 1.0 & $2(4)$ \\
\hline 1.5 & $7(13)$ \\
\hline 2.0 & $10(19)$ \\
\hline 2.5 & $6(11)$ \\
\hline 3.0 & $10(19)$ \\
\hline 3.5 & $1(2)$ \\
\hline 4.0 & $11(20)$ \\
\hline 4.5 & $1(2)$ \\
\hline Not available & $5(9)$ \\
\hline \multicolumn{2}{|l|}{ Primary cancer type } \\
\hline Melanoma & $17(31)$ \\
\hline Non-small cell lung cancer & $20(37)$ \\
\hline Renal cell carcinoma & $4(7)$ \\
\hline Breast cancer & $4(7)$ \\
\hline Colon cancer & $2(4)$ \\
\hline Small cell lung cancer & $1(2)$ \\
\hline Other & $6(11)$ \\
\hline Extracranial disease & $37(69)$ \\
\hline Chemotherapy/immunotherapy in the past mo & $28(52)$ \\
\hline Immunotherapy & $13(24)$ \\
\hline Chemotherapy & $15(28)$ \\
\hline
\end{tabular}


» CONTINUED FROM PAGE 1793

TABLE 1. Demographic and clinical characteristics of the study patients

\begin{tabular}{lc}
\multicolumn{1}{c}{ Characteristic } & Value \\
\hline Chemotherapy/immunotherapy $>1$ mo & $22(41)$ \\
\hline Prior resection & $24(44)$ \\
\hline Upfront WBRT & $6(11)$ \\
No. of fractions & 10 \\
Total WBRT dose, Gy & 30 \\
\hline
\end{tabular}

Values are presented as the number of patients (\%) unless stated otherwise. Mean values are presented as mean $\pm S D$, and median values are presented as median (range).

died and additional GKRS was performed in $49 \%$ of the 109 cases for intracranial disease progression. At the last available imaging follow-up, the majority (58\%) of treated metastatic tumors were stable or decreased in size, and $4.6 \%$ of the treated lesions were categorized as local failure.

\section{Quality of Life}

Fifty-four patients completed the EQ-5D questionnaire before GKRS and at least once after GKRS, and $80 \%$ of these patients also completed the EQ-5D at least twice after GKRS (Table 4). The first post-SRS visit was at a median of 2.59 months, and the final post-SRS visit was at 14.72 months after the SRS. Patients with 2 or more post-

TABLE 2. GKRS treatment characteristics

\begin{tabular}{lc}
\hline & Value \\
\hline Lesions treated at each GKRS session & \\
Mean no. & $3.45 \pm 3.27$ \\
Median no. & $2(1-14)$ \\
1 & $43(39)$ \\
$2-3$ & $28(26)$ \\
$4-5$ & $16(15)$ \\
$>5$ & $22(20)$ \\
\hline Lesion location per treatment & \\
Frontal & $67(61)$ \\
Parietal & $30(28)$ \\
Occipital & $31(28)$ \\
Temporal & $34(31)$ \\
Cerebellar & $38(35)$ \\
Basal ganglia & $7(6)$ \\
Brainstem & $12(11)$ \\
\hline Total vol of tumor treated per GKRS session, $\mathrm{cm}^{3}$ & \\
Mean & $3.38 \pm 4.98$ \\
Median & $1.17(0.07-20.27)$ \\
\hline Prescription dose, Gy & $18.85 \pm 3.55$ \\
Mean & $18(12-32)$ \\
Median &
\end{tabular}

Values are presented as the number of procedures (\%) unless stated otherwise. Mean values are presented as mean $\pm S D$, and median values are presented as median (range).
TABLE 3. Clinical outcomes

\begin{tabular}{lc}
\hline & Value \\
\hline Length of clinical follow-up (mos) & \\
Mean & $17.02 \pm 12.05$ \\
Median & $12.95(2.23-45.15)$ \\
\hline Repeat GKRS & $53(49)$ \\
\hline Additional WBRT & $1(1)$ \\
\hline Additional resection & $1(1)$ \\
\hline Mortality, no. of patients (\%) & $13(24)$ \\
\hline Length of imaging follow-up (mos) & \\
Mean & $14.34 \pm 14.52$ \\
Median & $9.97(1-101)$ \\
\hline Imaging response & \\
Stable or decrease & $63(58)$ \\
Progression & $39(36)$ \\
Data not available & $7(6)$ \\
\hline
\end{tabular}

Values are presented as the number of procedures (\%) unless stated otherwise. Mean values are presented as mean \pm SD, and median values are presented as median (range).

SRS visits when compared with patients with 1 post-SRS visit had higher pre-SRS EQ-5D index scores $(p=0.05)$, while age $(\mathrm{p}=0.52)$ and number of treated lesions $(\mathrm{p}=$ 0.14 ) were similar.

The proportion of patients reporting some problems on the EQ-5D self-care subscale progressively increased from the pre-SRS to first and last post-SRS follow-up visits $(9 \%, 11 \%$, and $18 \%$, respectively, $p=0.03$; Table 4$)$. The EQ-5D index score and proportion of patients reporting perceived difficulties on other EQ-5D dimensions remained stable during the follow-up period ( $p \geq 0.106$ for all) (Table 4 and Fig. 1). The proportion of patients with a clinically meaningful change in the EQ-5D index score at the first and last post-SRS relative to pre-SRS levels was not statically significantly different $(\mathrm{p}=0.052)$ (Table 4$)$.

Next, we investigated clinical and demographic predictors of worsening of the EQ-5D index score and subscale scores at the last post-SRS visit when compared with preSRS assessments (Table 5). A higher RPA class (i.e., worse prognostic category) was associated with greater odds for EQ-5D index score deterioration at the last post-SRS visit, independently from other considered predictors (OR 4.791, 95\% CI 1.00-22.946; $\mathrm{p}=0.050$ ). Upfront WBRT was independently associated with an increased likelihood of deterioration in the EQ-5D self-care (OR 21.54, 95\% CI $1.33-348.13, \mathrm{p}=0.03$ ) and usual activities (OR 14.50, 95\% CI 1.415-148.57; $p=0.024$ ) dimensions. A greater number of lesions predicted greater odds of deterioration in the EQ-5D anxiety/depression dimension (OR 2.041, 95\% CI $1.202-3.545 ; \mathrm{p}=0.008)$.

In Kaplan-Meier analyses, survival was significantly shorter for patients who had lower pre-SRS EQ-5D index scores $(\leq 0.84)$ when compared with patients who had higher $(>0.84)$ EQ-5D index scores $(21.8 \pm 3.8$ vs $37.1 \pm$ 3.1 months; log-rank test $=6.581, \mathrm{p}=0.01$ [Fig. 2]). A low pre-SRS EQ-5D score remained associated with a shorter overall survival (OR 18.956, 95\% CI 2.793-128.64; $\mathrm{p}=$ 
TABLE 4. QOL before GKRS and at follow-up

\begin{tabular}{|c|c|c|c|c|}
\hline & Before GKRS & 1st Follow-Up & Last Follow-Up & $p$ Value \\
\hline No. of patients at risk & 54 & 54 & $44^{*}$ & \\
\hline Median interval after GKRS, mos & & $2.59(0.13-21.08)$ & $14.72(2.52-45.21)$ & \\
\hline \multicolumn{5}{|l|}{ EQ-5D dimensions } \\
\hline Mobility & & & & 0.695 \\
\hline No problems & $38(70)$ & $40(74)$ & $31(70)$ & \\
\hline Some problems & $16(30)$ & $14(26)$ & $13(30)$ & \\
\hline Extreme problems & $0(0)^{\prime}$ & $0(0)$ & $0(0)$ & \\
\hline Self-care & & & & 0.03 \\
\hline No problems & $49(91)$ & $48(89)$ & $36(82)$ & \\
\hline Some problems & $5(9)$ & $6(11)$ & $8(18)$ & \\
\hline Extreme problems & $0(0)$ & $0(0)$ & $0(0)$ & \\
\hline Usual activities & & & & 0.128 \\
\hline No problems & $38(70)$ & $36(67)$ & $25(57)$ & \\
\hline Some problems & $16(30)$ & $17(31)$ & $19(43)$ & \\
\hline Extreme problems & $0(0)$ & $1(2)$ & $0(0)$ & \\
\hline Pain/discomfort & & & & 0.106 \\
\hline No problems & $37(69)$ & $36(67)$ & $27(61)$ & \\
\hline Some problems & $17(31)$ & $18(33)$ & $14(32)$ & \\
\hline Extreme problems & $0(0)$ & $0(0)$ & $3(7)$ & \\
\hline Anxiety/depression & & & & 0.857 \\
\hline No problems & $33(61)$ & $35(65)$ & $25(58)$ & \\
\hline Some problems & $18(33)$ & $18(33)$ & $17(40)$ & \\
\hline Extreme problems & $3(6)$ & $1(2)$ & $1(2)$ & \\
\hline Median EQ-5D index score & $0.854(0.55-1.00)$ & $0.844(0.44-1.00)$ & $0.843(0.27-1.00)$ & 0.539 \\
\hline EQ-5D index MCID† & & & & 0.052 \\
\hline Improvement & & $17(32)$ & $10(19)$ & \\
\hline No change & & $27(50)$ & $19(35)$ & \\
\hline Worsening & & 10 (19) & $14(26)$ & \\
\hline
\end{tabular}

$\mathrm{MCID}=$ minimal clinically important difference.

Values are presented as the number of patients (\%) unless stated otherwise. Median values are presented as median (range). Boldface type indicates statistical significance.

* Information was missing for 1 patient in the anxiety/depression dimension.

†EQ-5D-3L index score change from pre-GKRS: > 0.1 points, improvement; from -0.1 to 0.1 points, no change; and $<-0.1$ points, worsening.

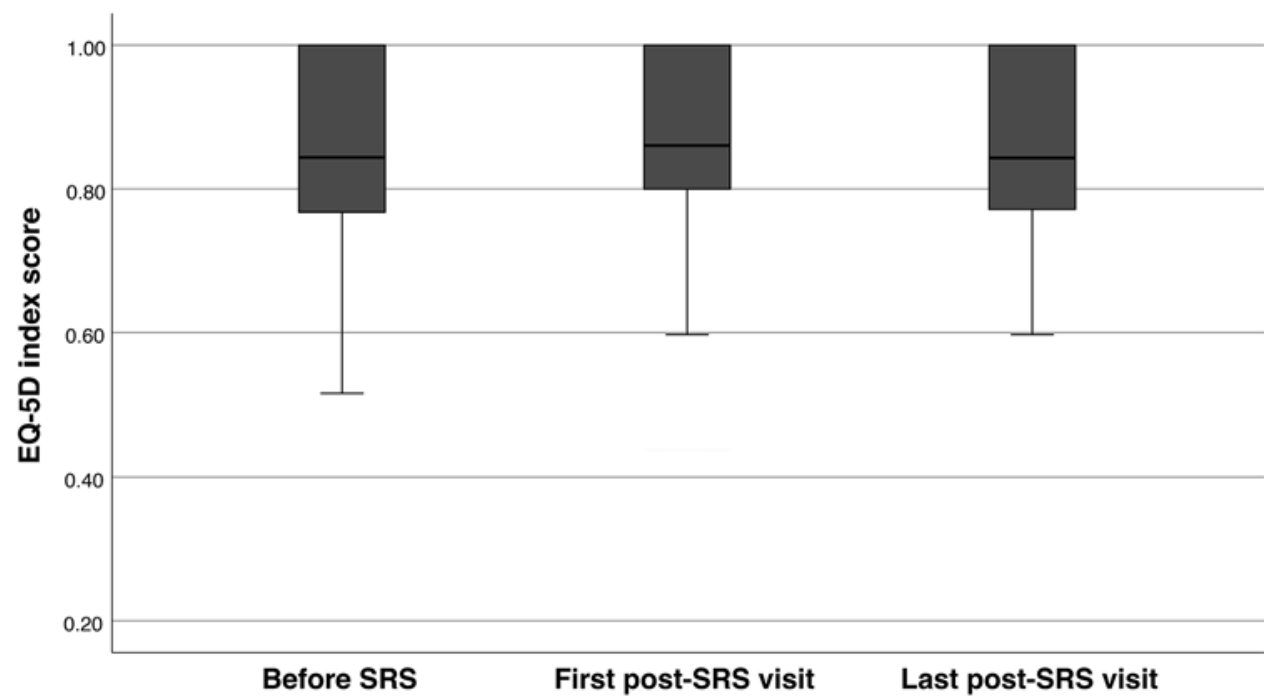

FIG. 1. EQ-5D index score before SRS and at the first and last post-SRS visits $(p=0.539)$. 
TABLE 5. Predictors of QOL decline at last post-SRS follow-up visit*

\begin{tabular}{|c|c|c|c|c|c|c|}
\hline & \multicolumn{6}{|c|}{ EQ-5D Dimensions (deterioration) } \\
\hline & Index Score & Mobility & Self-Care & Usual Activities & Pain/Discomfort & Anxiety/Depression \\
\hline RPA class & $\begin{array}{c}4.791(1.00-22.946) \\
0.050\end{array}$ & 0.321 & 0.314 & 0.457 & 0.365 & 0.063 \\
\hline No. of lesions & 0.207 & 0.570 & 0.356 & 0.645 & 0.689 & $\begin{array}{c}2.041(1.202-3.545) \\
0.008\end{array}$ \\
\hline Prior WBRT & 0.055 & 0.234 & $\begin{array}{c}21.539(1.33-348.13) \\
0.031\end{array}$ & $\begin{array}{c}14.50(1.415-148.57), \\
0.024\end{array}$ & 0.162 & 0.156 \\
\hline KPS score & 0.764 & 0.299 & 0.076 & 0.785 & 0.621 & 0.177 \\
\hline Age & 0.101 & 0.405 & 0.541 & 0.804 & 0.718 & 0.986 \\
\hline
\end{tabular}

KPS = Karnofsky Performance Scale.

ORs are presented for all variables. For the independent predictors, $95 \% \mathrm{Cls}$ are given, followed by the $\mathrm{p}$ value.

${ }^{*}$ Multivariate binary regression analysis adjusted for patient age, RPA class, KPS score, number of lesions, and prior WBRT.

$0.003)$, greater mortality risk at 1 year (OR $37.134,95 \%$ CI $3.754-367.344 ; \mathrm{p}=0.02$ ), and at 2 years (OR $11.341,95 \%$ CI 2.537-50.691; $\mathrm{p}=0.001$ ) after SRS in Cox regression analyses adjusted for clinical and demographic variables.

\section{Discussion}

We found that QOL was typically preserved in brain metastasis patients treated with SRS. Clinically meaningful worsening of the EQ-5D index was reported by $19 \%$ of patients at the first post-SRS visit and by $26 \%$ of patients at the last post-SRS assessment. A higher RPA class was associated with deterioration in the EQ-5D index score, upfront WBRT predicted deterioration of self-care and usual activities, and a greater number of lesions predicted deterioration of mental health. Worse pre-SRS QOL was associated with shorter survival after SRS when adjusting for clinical and demographic variables.
We found that SRS for metastatic brain lesions was associated with preservation of QOL and perceived level of mobility, usual activities, pain, and anxiety/depression during a median follow-up of 14 months (range 2.5-45.2 months). The proportion of patients reporting perceived difficulties with self-care increased during the course of the study. At the individual patient level, the majority of patients reported stable QOL during the course of the follow-up, while 19\% of patients reported improvement and $26 \%$ of patients reported worsening of their QOL at the last post-SRS visit. Increased perceived difficulties in self-care can be partially explained by systemic disease progression. Our findings largely agree with those of prior studies documenting that QOL is often preserved after

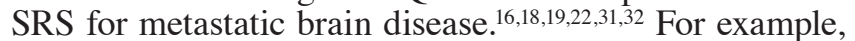
a prospective study by Skeie and colleagues included 97 consecutive patients treated with GKRS for 1 to 6 brain metastases and found that scores on the Functional As-

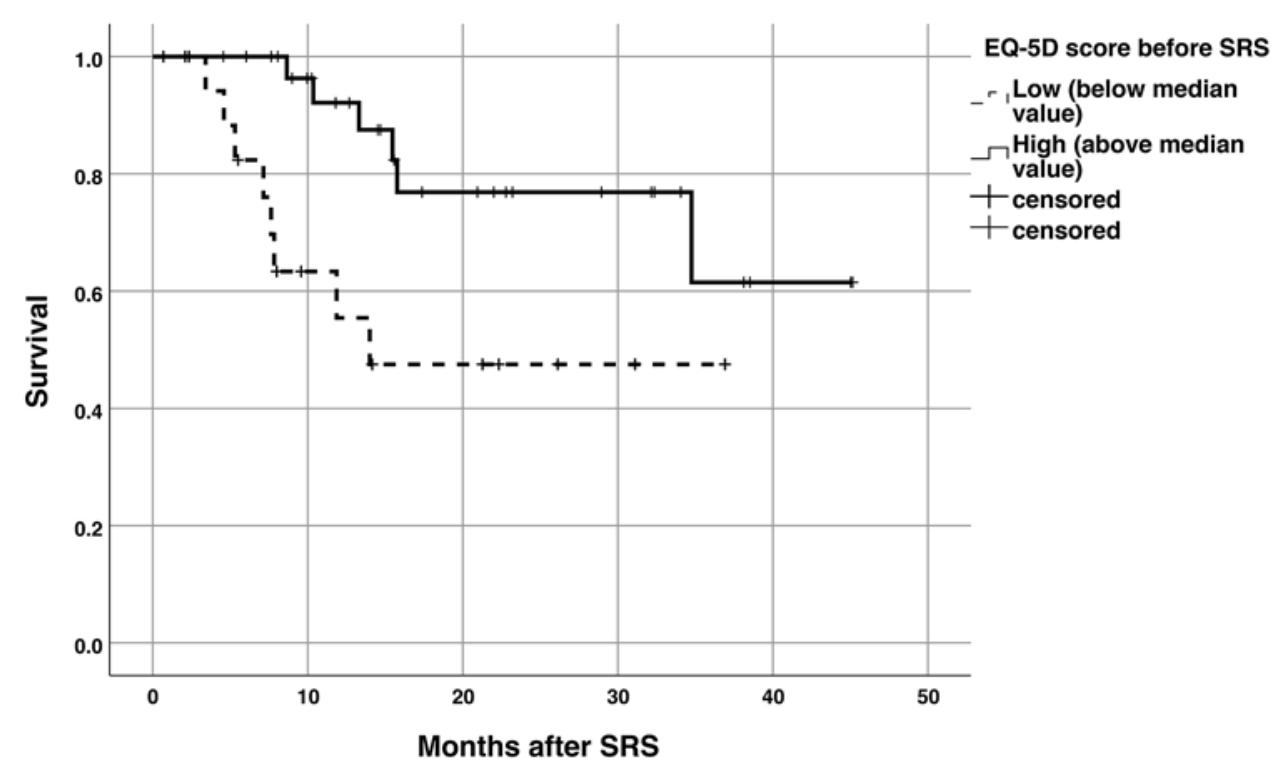

FIG. 2. Kaplan-Meier curve showing the association of the pre-SRS score with overall survival after SRS. 
sessment of Cancer Therapy-Brain (FACT-BR) questionnaire dimensions remained preserved during the 12-month follow-up after SRS. ${ }^{19}$ Another prospective study in 97 patients with brain metastases treated with a single fraction or fractionated SRS and followed for 6 months, found that QOL was largely preserved across QOL dimensions included in cancer and brain tumor-specific European Organization for Research and Treatment of Cancer (EORTC) QLQ-C30 and BN20 questionnaires. ${ }^{31}$ The authors noted deterioration of only physical functioning and fatigue QOL domains during the course of their study. However, others found a decline in QOL after SRS for brain metastases. ${ }^{17,33,34}$ For example, a study from the Cleveland Clinic that included 67 patients who received 122 GKRS treatments for metastatic brain lesions reported deterioration of all EQ-5D subscores at the last post-SRS follow-up visit at 12 months (range 4-25 months). ${ }^{17,33}$ Another study in geriatric brain metastasis patients treated with GKRS also reported a decline of the Spitzer QOL index at 6 months and at 12 months after SRS. ${ }^{34}$ While some groups noted progressive decline after SRS for brain metastases at a group level, results at the individual patient level remain mixed, with patients reporting stable QOL or worsening or improvement of their QOL during a longterm follow-up, suggesting between-patient variability of QOL trajectories during the course of cancer treatment. The observed differences between studies of QOL trajectories at a group level may be attributed to differences in study inclusion criteria (disease severity, age, and prior and current therapies) and study design (QOL questionnaires and assessment intervals). Nevertheless, there appears to be compelling evidence that SRS allows preservation or even improvement of QOL in the majority of brain metastasis patients. Given individual variation of post-SRS QOL trajectories, monitoring of QOL can provide valuable information that can be used for optimized care of cancer patients with brain metastases.

At the last follow-up visit, $43 \%, 42 \%$, and $32 \%$ of patients reported some or extreme problems with usual activities, anxiety/depression, and pain/discomfort, respectively, indicating that these factors are important sources of QOL impairment in the long-term cancer survivors treated with SRS. Indeed, neurobehavioral symptoms such as depression often remain undiagnosed and therefore unor undertreated in cancer patients. ${ }^{35,36}$ Neurobehavioral symptoms and functional concerns can be identified using QOL instruments and should be addressed in cancer patients in order to optimize preservation of their QOL. ${ }^{37}$ The use of standardized instruments for assessment of QOL of cancer patients receiving SRS should be administered to better identify the major sources of impairment that should be addressed.

Clinically meaningful decline of EQ-5D index at the last post-SRS visit was independently predicted by RPA class. Upfront WBRT and a greater number of lesions were independently associated with deterioration of self-care and usual activities, and mental health, respectively. These findings indicate that patients with poor functional status and significant disease burden should be considered at elevated risk for deterioration of QOL. Furthermore, upfront WBRT should be considered a risk factor for deteriora- tion of perceived functional aspects of QOL. Prior studies have shown that WBRT for brain metastases can lead to worse cognitive outcomes and QOL. For example, a phase III clinical trial by the EORTC group that randomized 359 patients with 1 to 3 brain metastases to either WBRT or observation after resection or radiosurgery found that in the WBRT arm when compared with the observation arm, the QOL was significantly worse for global health status at 9 months, physical functioning at 8 weeks, cognitive functioning at 12 months, and fatigue at 8 weeks. ${ }^{10}$ The N0574 trial found that among patients with 1 to 3 brain metastases, the use of SRS only when compared with SRS plus WBRT was associated with less cognitive deterioration at 3 months. ${ }^{38}$ Another prospective trial by Chang and colleagues randomized 58 brain metastases patients to either SRS alone $(n=30)$ or SRS plus WBRT $(n=28)$ and found that patients treated with SRS plus WBRT were at elevated risk for decline in cognitive functioning by 4 months when compared with the SRS-only group. ${ }^{9}$ However, in the later study, the differences in FACT-BR score were not significant between the two groups. In our series, QOL was preserved in the majority of patients treated with SRS, and WBRT emerged as an important predictor of QOL decline, hence echoing prior randomized studies comparing SRS only with SRS plus WBRT. History of WBRT can be an important and independent predictor of QOL decline after SRS, and this observation should be considered, given the increasing survival of cancer patients.

Worse pre-SRS was independently associated with shorter survival after SRS and with greater mortality risk at 1 and 2 years after SRS. These findings contribute to the growing body of evidence documenting that QOL is an important predictor of survival of cancer patients. ${ }^{39}$ For example, a study in 269 brain metastases patients treated with WBRT found that lower pre-WBRT QOL was associated with shorter survival. ${ }^{40}$ QOL is an important outcome measure of cancer treatment and is recommended for treatment guidance of cancer patients ${ }^{13-15}$ However, the prognostic importance of pre-SRS QOL for cancer patients remains understudied. Our findings suggest that monitoring of QOL carries prognostic value in the management of brain metastasis patients treated with SRS and therefore should be implemented.

\section{Limitations}

Our study has limitations. First, QOL was evaluated using the EQ-5D questionnaire, which is a generic and a not brain tumor- or cancer-specific QOL instrument. However, the EQ-5D is widely used to study QOL in brain metastasis patients treated with SRS ${ }^{17,18,33}$ and in non-CNS cancer patients. ${ }^{28} \mathrm{EQ}-5 \mathrm{D}$ is also routinely integrated into a lot of NeuroPoint Alliance registries. Brain tumor-specific QOL scales, such as the EORTC BM-22 and FACT$\mathrm{BR}$, were specifically designed to capture brain tumor-related symptom burden and are commonly used in research studies. However, due to a greater number of items, their use might require greater personnel and time resources, and this can preclude their use in everyday neurooncology practice. Optimal and clinically feasible QOL monitoring strategies after SRS remain to be elucidated. Also, not all patients completed all follow-up visits, which can 
be attributed to clinical status deterioration preventing the patient from completion of the questionnaire and showing up for a follow-up visit. Furthermore, the moderate sample size limited the statistical power of our study. Finally, this single-institution experience places our results at risk for selection bias; therefore, multicenter efforts aiming to establish QOL trajectories after SRS for brain metastases are strongly encouraged. ${ }^{18}$ Given patient inclusion criteria, our results should not be generalized to brain metastasis patients treated with non-SRS treatment modalities. A NeuroPoint Alliance SRS registry analysis should be undertaken to better assess a larger cohort of data; this effort will likely require analysis accustomed to working with big data initiatives.

\section{Conclusions}

QOL is largely preserved in brain metastasis patients treated with SRS, with the majority of patients reporting stable or improved QOL. A higher RPA class is independently associated with QOL deterioration. Upfront WBRT is an independent predictor of functional status (self-care and usual activities) decline, while a greater number of lesions predicts mental health decline. Worse pre-SRS QOL was associated with shorter survival after SRS, adjusting for clinical and demographic variables. Monitoring of QOL should be considered in cancer patients treated with SRS for brain metastases. However, optimal QOL monitoring strategies remain to be identified.

\section{References}

1. Nayak L, Lee EQ, Wen PY. Epidemiology of brain metastases. Curr Oncol Rep. 2012;14(1):48-54.

2. Butowski NA. Epidemiology and diagnosis of brain tumors. Continuum (Minneap Minn). 2015;21(2 Neuro-oncology): 301-313.

3. Davis FG, Dolecek TA, McCarthy BJ, Villano JL. Toward determining the lifetime occurrence of metastatic brain tumors estimated from 2007 United States cancer incidence data. Neuro Oncol. 2012;14(9):1171-1177.

4. Wen PY, Loeffler JS. Management of brain metastases. Oncol (Williston Park). 1999;13(7):941-954, 957-962, 9.

5. Lin X, DeAngelis LM. Treatment of brain metastases. J Clin Oncol. 2015;33(30):3475-3484.

6. Soffietti R, Cornu P, Delattre JY, et al. EFNS Guidelines on diagnosis and treatment of brain metastases: report of an EFNS Task Force. Eur J Neurol. 2006;13(7):674-681.

7. Churilla TM, Ballman KV, Brown PD, et al. Stereotactic radiosurgery with or without whole-brain radiation therapy for limited brain metastases: A secondary analysis of the North Central Cancer Treatment Group N0574 (Alliance) Randomized Controlled Trial. Int J Radiat Oncol Biol Phys. 2017; 99(5):1173-1178.

8. Kayama T, Sato S, Sakurada K, et al. Effects of surgery with salvage stereotactic radiosurgery versus surgery with wholebrain radiation therapy in patients with one to four brain metastases (JCOG0504): A phase III, noninferiority, randomized controlled trial. J Clin Oncol. 2018;20:JCO2018786186.

9. Chang EL, Wefel JS, Hess KR, et al. Neurocognition in patients with brain metastases treated with radiosurgery or radiosurgery plus whole-brain irradiation: a randomised controlled trial. Lancet Oncol. 2009;10(11):1037-1044.

10. Soffietti R, Kocher M, Abacioglu UM, et al. A European Organisation for Research and Treatment of Cancer phase III trial of adjuvant whole-brain radiotherapy versus observation in patients with one to three brain metastases from solid tumors after surgical resection or radiosurgery: quality-of-life results. J Clin Oncol. 2013;31(1):65-72.

11. Yamamoto M, Serizawa T, Higuchi Y, et al. A multiinstitutional prospective observational study of stereotactic radiosurgery for patients with multiple brain metastases (JLGK0901 Study Update): Irradiation-related complications and long-term maintenance of Mini-Mental State Examination scores. Int J Radiat Oncol Biol Phys. 2017;99(1):31-40.

12. Mauer M, Stupp R, Taphoorn MJB, et al. The prognostic value of health-related quality-of-life data in predicting survival in glioblastoma cancer patients: results from an international randomised phase III EORTC Brain Tumour and Radiation Oncology Groups, and NCIC Clinical Trials Group study. $\mathrm{Br}$ J Cancer. 2007;97(3):302-307.

13. Bottomley A, Pe M, Sloan J, et al. Analysing data from patient-reported outcome and quality of life endpoints for cancer clinical trials: a start in setting international standards. Lancet Oncol. 2016;17(11):e510-e514.

14. Ediebah DE, Quinten C, Coens C, et al. Quality of life as a prognostic indicator of survival: A pooled analysis of individual patient data from Canadian cancer trials group clinical trials. Cancer. 2018;124(16):3409-3416.

15. Quinten C, Coens C, Mauer M, et al. Baseline quality of life as a prognostic indicator of survival: a meta-analysis of individual patient data from EORTC clinical trials. Lancet Oncol. 2009;10(9):865-871.

16. Bragstad S, Flatebø M, Natvig GK, et al. Predictors of quality of life and survival following Gamma Knife surgery for lung cancer brain metastases: a prospective study. J Neurosurg. 2018;129(1):71-83.

17. Miller JA, Kotecha R, Barnett GH, et al. Quality of life following stereotactic radiosurgery for single and multiple brain metastases. Neurosurgery. 2017;81(1):147-155.

18. Sheehan JP, Grills I, Chiang VL, et al. Quality of life outcomes for brain metastasis patients treated with stereotactic radiosurgery: pre-procedural predictive factors from a prospective national registry. J Neurosurg. 2018;131(6):1848-1854.

19. Skeie BS, Eide GE, Flatebø M, et al. Quality of life is maintained using Gamma Knife radiosurgery: a prospective study of a brain metastases patient cohort. J Neurosurg. 2017; 126(3):708-725.

20. Burns TC, Awad AJ, Li MD, Grant GA. Radiation-induced brain injury: low-hanging fruit for neuroregeneration. Neurosurg Focus. 2016;40(5):E3.

21. Greene-Schloesser D, Robbins ME, Peiffer AM, et al. Radiation-induced brain injury: a review. Front Oncol. 2012;2:73.

22. Verhaak E, Gehring K, Hanssens PEJ, et al. Health-related quality of life in adult patients with brain metastases after stereotactic radiosurgery: a systematic, narrative review. Support Care Cancer. 2020;28(2):473-484.

23. Sheehan JP, Kavanagh BD, Asher A, Harbaugh RE. Inception of a national multidisciplinary registry for stereotactic radiosurgery. J Neurosurg. 2016;124(1):155-162.

24. Sperduto PW, Kased N, Roberge D, et al. Summary report on the graded prognostic assessment: an accurate and facile diagnosis-specific tool to estimate survival for patients with brain metastases. J Clin Oncol. 2012;30(4):419-425.

25. Gaspar L, Scott C, Rotman M, et al. Recursive partitioning analysis (RPA) of prognostic factors in three Radiation Therapy Oncology Group (RTOG) brain metastases trials. Int J Radiat Oncol Biol Phys. 1997;37(4):745-751.

26. Snell JW, Sheehan J, Stroila M, Steiner L. Assessment of imaging studies used with radiosurgery: a volumetric algorithm and an estimation of its error. Technical note. J Neurosurg. 2006;104(1):157-162.

27. Pickard AS, Wilke CT, Lin H-W, Lloyd A. Health utilities using the EQ-5D in studies of cancer. Pharmacoeconomics. 2007;25(5):365-384. 
28. Schwenkglenks M, Matter-Walstra K. Is the EQ-5D suitable for use in oncology? An overview of the literature and recent developments. Expert Rev Pharmacoecon Outcomes Res. 2016;16(2):207-219.

29. Kvam AK, Fayers PM, Wisloff F. Responsiveness and minimal important score differences in quality-of-life questionnaires: a comparison of the EORTC QLQ-C30 cancer-specific questionnaire to the generic utility questionnaires EQ-5D and 15D in patients with multiple myeloma. Eur J Haematol. 2011;87(4):330-337.

30. Salvetti DJ, Nagaraja TG, McNeill IT, et al. Gamma Knife surgery for the treatment of 5 to 15 metastases to the brain: clinical article. J Neurosurg. 2013;118(6):1250-1257.

31. Habets EJJ, Dirven L, Wiggenraad RG, et al. Neurocognitive functioning and health-related quality of life in patients treated with stereotactic radiotherapy for brain metastases: a prospective study. Neuro Oncol. 2016;18(3):435-444.

32. van der Meer PB, Habets EJJ, Wiggenraad RG, et al. Individual changes in neurocognitive functioning and health-related quality of life in patients with brain oligometastases treated with stereotactic radiotherapy. J Neurooncol. 2018;139(2): 359-368.

33. Kotecha R, Damico N, Miller JA, et al. Three or more courses of stereotactic radiosurgery for patients with multiply recurrent brain metastases. Neurosurgery. 2017;80(6):871-879.

34. Randolph DM, McTyre E, Klepin H, et al. Impact of radiosurgical management of geriatric patients with brain metastases: clinical and quality of life outcomes. J Radiosurg SBRT. 2017;5(1):35-42.

35. Caruso R, Nanni MG, Riba M, et al. Depressive spectrum disorders in cancer: prevalence, risk factors and screening for depression: a critical review. Acta Oncol. 2017;56(2):146-155.

36. Pranckeviciene A, Bunevicius A. Depression screening in patients with brain tumors: a review. CNS Oncol. 2015;4(2): 71-78.

37. Bunevicius A. Reliability and validity of the SF-36 Health Survey Questionnaire in patients with brain tumors: a crosssectional study. Health Qual Life Outcomes. 2017;15(1):92.
38. Brown PD, Jaeckle K, Ballman KV, et al. Effect of radiosurgery alone vs radiosurgery with whole brain radiation therapy on cognitive function in patients with 1 to 3 brain metastases: a randomized clinical trial. JAMA. 2016;316(4):401-409.

39. Montazeri A. Quality of life data as prognostic indicators of survival in cancer patients: an overview of the literature from 1982 to 2008. Health Qual Life Outcomes. 2009;7:102.

40. Wong E, Rowbottom L, Tsao M, et al. Prognostic value of pre- and post-treatment health-related quality of life in predicting survival of patients with brain metastases. CNS Oncol. 2017;6(2):119-129.

\section{Disclosures}

The authors report no conflict of interest concerning the materials or methods used in this study or the findings specified in this paper.

\section{Author Contributions}

Conception and design: Sheehan, Bunevicius. Acquisition of data: all authors. Analysis and interpretation of data: Sheehan, Bunevicius. Drafting the article: Bunevicius. Critically revising the article: all authors. Reviewed submitted version of manuscript: Sheehan, Bunevicius, Lavezzo, Shabo. Approved the final version of the manuscript on behalf of all authors: Sheehan. Statistical analysis: Bunevicius. Administrative/technical/material support: Sheehan. Study supervision: Sheehan.

\section{Correspondence}

Jason P. Sheehan: University of Virginia Health System, Charlottesville, VA. jps2f@hscmail.mcc.virginia.edu. 